\title{
A study on the asymmetric cylinder wall thickness difference discrimination by dolphins
}

\author{
Wen Feng and Yu Zhang ${ }^{\text {a) }}$ \\ Key Laboratory of Underwater Acoustic Communication and Marine Information Technology of the Ministry \\ of Education, College of Ocean and Earth Sciences, Xiamen University, Xiamen 361005, \\ People's Republic of China \\ Chong Wei \\ Acoustic Research Laboratory, Tropical Marine Science Institute, National University of Singapore, \\ 12A Kent Ridge Road, Singapore, 119222, Singapore
}

(Received 1 March 2018; revised 2 August 2018; accepted 3 August 2018; published online 27 August 2018)

Atlantic bottlenose dolphins (Tursiops truncatus) can effectively discriminate between water-filled cylinders with different wall thicknesses. The dolphins' performance may be particularly good when the cylinders are thinner. The dolphins' performance is also asymmetric, in that the discrimination accuracy is not equal when the target thickness increases and decreases by the same amount. Inspired by this, a finite element model is proposed here to mimic a dolphin actively transmitting sound to discriminate between targets using acoustic echoes. The waveforms and frequency spectra of acoustic echoes from a standard cylinder and comparison cylinders with wall thickness differences of $\pm 0.3 \mathrm{~mm}$ were compared. The employed model simulations show good agreement with previous experimental measurements by Au and Pawloski [(1992). J. Compar. Physiol. A 170(1), 41-47]. Asymmetric arrival time shifts were found for the echo peaks and troughs with the same sequence numbers when the wall thicknesses were increased and decreased by the same amount. This asymmetry became more significant for echo peaks and troughs with higher sequence numbers. Apart from these asymmetric arrival time shifts of the acoustic echoes, the patterns of echo waveforms, the spatial distributions of sound pressures in the water, and the particle vibratory displacements in the cylinders were also found to vary with cylinder thickness. The physical origin of this asymmetric discrimination by the dolphins was explored using both geometric acoustics and wave acoustics. The asymmetry observed might be caused by the circumferential surface (Lamb) wave in the cylinder wall, which is a wave acoustics phenomenon that cannot be derived from geometric acoustics. The findings in this paper might be valuable not just for understanding the mechanism of the effect described, but also for helping the development of biomimetic intelligence for robust signal processing in underwater target discrimination. (c) 2018 Acoustical Society of America. https://doi.org/10.1121/1.5051330

[WWA]

Pages: 1018-1027

\section{INTRODUCTION}

Marine Corps forces have relied in the past on highly trained dolphins to detect mines and avoid obstacles in shallow water, where the performance of dolphin sonar was superior to that of technical sonar (Moore, 1997). Cetacean echolocation is superior to passive audition because actively transmitting directional sounds gives better perception of the surrounding environment. As a result of long-term evolution and selection, the cetacean head structure guarantees optimum transmission and reception of sounds, and the brain processes echoes from the surrounding environment, where targets of interest may exist. Previous researchers have examined the effects of the experimental conditions on the dolphin capabilities, such as varying the range and angle from the target to the dolphin or by adding interference such

\footnotetext{
a) Also at: Department of Mechanical Engineering, Massachusetts Institute of Technology, Cambridge, MA 02139, USA. Electronic mail: yuzhang@xmu.edu.cn
}

as noise and reverberations (Au and Snyder, 1980; $\mathrm{Au}$ and Turl, 1983; Turl et al., 1987; Turl et al., 1991; Helweg et al., 1996; Moore et al., 2008; Wezensky et al., 2011). In target discrimination experiments, animals normally compared between two targets with only one difference in features like size, shape, structure, and material (Busnel and Fish, 1980; Au and Turl, 1991; Brill et al., 1992; Au and Pawloski, 1992; Pack and Herman, 1995; Zaitseva and Korolev, 2005). In oceanic ambient noise, one bottlenose dolphin was found to have detection threshold between 7.5 and $9.8 \mathrm{~dB}$ in terms of echo energy to noise spectral density (Au and Hastings, 2008). Au and Penner (1981) used a bottlenose dolphin to show that the accuracy of target detection was almost linear with the echo-to-reverberation ratio and its $50 \%$ correct detection occurred at an energy echo-to-reverberation ratio of approximately $0.25 \mathrm{~dB}$. Moore et al. (2008) found that a bottlenose dolphin has angular target detection thresholds of 26 degrees left and 21 degrees right for a spherical target and 19 degrees left and 13 degrees right for a cylindrical target at a distance of $9 \mathrm{~m}$. One bottlenose dolphin could 
identify a wall thickness difference of $0.2 \mathrm{~mm}$ with $75 \%$ accuracy when the two cylinders were both $50 \mathrm{~mm}$ in outer diameter and length and $5 \mathrm{~m}$ away from the dolphin ( $\mathrm{Au}$ and Hastings, 2008). Many researchers have tried to explain why these cetaceans have such amazing capabilities. The clues used by the dolphins were interpreted physically as ultrasonic spectroscopy techniques from which both peculiar time and frequency signatures were extracted (Gaunaurd et al., 1996). An acoustic basis for recognition of aspectdependent three-dimensional targets by an echolocating Atlantic bottlenose dolphin was investigated. The results showed that the dolphins can recognize the targets using a multidimensional representation containing amplitude and spectral information and can form stable representations of the targets based on echo characteristics (Helweg et al., 1996). Various neural networks were developed, trying to model the cognitive and perceptual processes in cetaceans (Au et al., 1995; Moore et al., 1993).

Exploring the mechanism of dolphin biosonar could provide inspiration for improving the performance of marine technical sonar (Müller and Kuc, 2007). For example, inspired by the ultrasound beam formations in dolphins (Wei et al., 2014; Song et al., 2016), a biomimetic projector with high subwavelength directivity was proposed to manipulate beams (Zhang et al., 2014; Gao et al., 2016). Under the excitation of a biomimetic click, the backscattering spectrum could be analyzed to classify cylindrical shells composed of different materials (Pailhas et al., 2010). Filtering out specular reflection, the maintained elastic component of the echoes from objects excited by a biomimetic dolphin click could provide better performance in target discrimination (Qiao et al., 2017).

Au and Pawloski (1992) have experimentally found an Atlantic bottlenose dolphin has an asymmetric cylinder wall thickness difference discrimination threshold of $-0.23 \mathrm{~mm}$ and $+0.27 \mathrm{~mm}$. More specifically, when the inner diameter increases or decreases by the same amount, with the outer diameter remaining constant, the discrimination accuracy of the dolphin is not equal or symmetric. This cannot be explained by geometric acoustics, where the arrival time shifts of the acoustic echoes should be symmetric, since dolphins are supposed to discriminate wall thickness differences by comparing arrival time delays between waves reflected from the front and rear walls. In addition, experimentally measured echoes are contaminated by noise from the water and electronic circuit systems. Therefore, in this paper, a finite element model without interference from measurement errors and ambient noise is proposed to investigate the asymmetric discrimination. The model mimics a dolphin actively transmitting sound to identify target differences using acoustic echoes. The broadband acoustic output of a bottlenose dolphin is represented by the dolphin click taken from $\mathrm{Au}$ and Pawloski (1992). The arrival time shifts of acoustic echoes for comparison cylinders with equal thickness increments of opposite sign were compared and analyzed. The spatial distributions of the sound pressure in water and the particle vibratory displacements in the cylinders were numerically derived.

\section{METHODS}

In the discrimination process, the clicks of the bottlenose dolphin interact with a water-filled aluminum cylindrical shell. When acoustic waves propagate within a liquid medium, the longitudinal waves can be described as

$$
\frac{1}{\rho_{0} c_{s}^{2}} \frac{\partial^{2} p}{\partial t^{2}}+\nabla \cdot\left(-\frac{1}{\rho_{0}} \nabla p\right)=0
$$

where $p$ is the sound pressure, $\rho_{0}$ is the density $\left(\mathrm{kg} / \mathrm{m}^{3}\right)$, and $c_{s}$ is the speed of sound $(\mathrm{m} / \mathrm{s})$. The density $\rho_{0}$ is included in the equation because of its variations in computation domain. When the acoustic waves interact with a solid object, the longitudinal and transverse waves can be described as

$$
\rho \frac{\partial^{2} \mathbf{u}}{\partial^{2} t}=(\lambda+2 \mu) \operatorname{grad}(\operatorname{divu})-\mu \operatorname{rot}(\operatorname{rotu}),
$$

where $\mathbf{u}$ is the position vector, $\lambda$ and $\mu$ are two Lame constants characterizing the compression and shear modulus in the solid, respectively. The boundary condition between the solid and liquid satisfies

$$
\begin{aligned}
& \mathbf{F}=-\mathbf{n}_{s} p, \\
& -\mathbf{n}_{\mathbf{a}} \cdot\left(-\frac{1}{\rho_{0}} \nabla p\right)=a_{n},
\end{aligned}
$$

where $\mathbf{F}$ is the boundary load (force/unit area) on solid, $\mathbf{n}_{s}$ is the outward-pointing unit normal vector seen from inside solid, $p$ is the acoustic pressure, $\mathbf{n}_{a}$ is the outward-pointing unit normal vector seen from inside liquid, $\rho_{0}$ is the density, and $a_{n}$ is normal acceleration of the solid surface in the liquid domain boundary.

To simulate the physical process described above, a two-dimensional underwater target detection model using a finite element method was developed, and is shown in Fig. 1. The finite element method has been widely used in acoustic field simulations to deal with problems such as irregular shapes, inhomogeneous distributions of the material, and complex boundary conditions. Considering the complex structure of an animal head, this numerical technique has been employed to investigate sound generation, beam formation and manipulation, and reception in cetaceans (Aroyan et al., 1992; Aroyan, 2001; Zhang et al., 2017). The COMSOL Multiphysics finite element modeling software (Stockholm, Sweden) was used to solve the finite element model. The parameters used in the model were given as: the density of water $\rho_{0}=998\left(\mathrm{~kg} / \mathrm{m}^{3}\right)$, the sound velocity in the water $c_{0}=1475(\mathrm{~m} / \mathrm{s})$, the density of aluminum $\rho_{1}=2700\left(\mathrm{~kg} / \mathrm{m}^{3}\right)$, the longitudinal sound velocity of aluminum $c_{l}=6420(\mathrm{~m} / \mathrm{s})$, and the transverse sound velocity of aluminum $c_{t}=3040(\mathrm{~m} / \mathrm{s})$. Figure 1 also presents the waveform and power spectrum of the broadband signal, which has a duration time of $60 \mu$ s and a peak frequency of $120 \mathrm{kHz}$ (Au and Pawloski, 1992). The cylinder was $1 \mathrm{~m}$ away from the sound source, whose radiated waves were incident broadside on the cylinder. The standard cylinder 


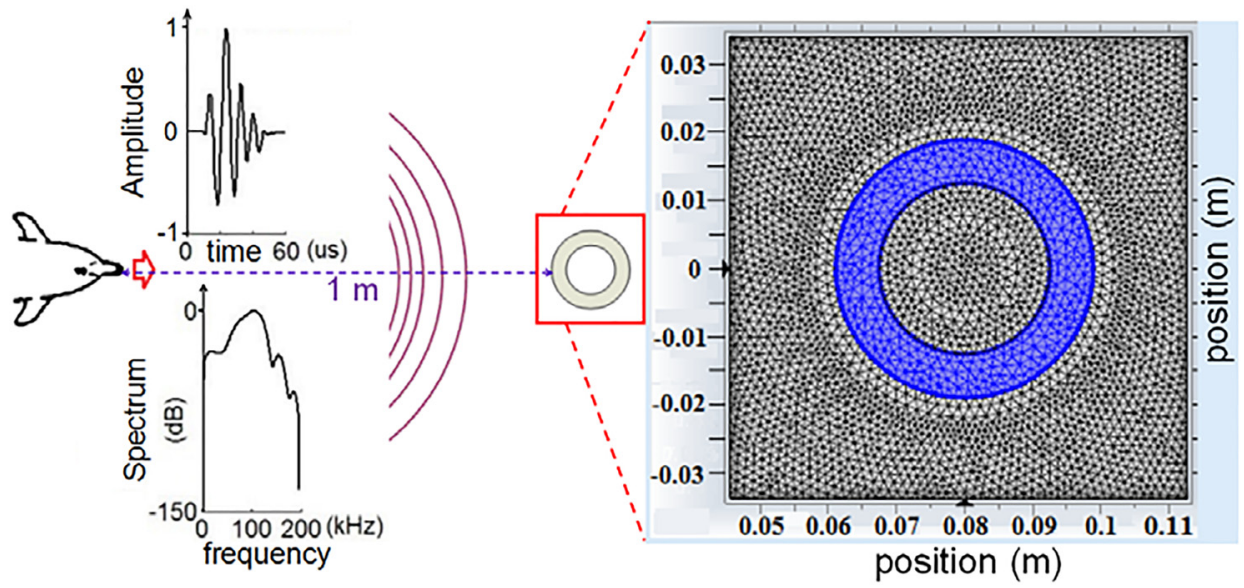

FIG. 1. (Color online) A systematic diagram of the underwater target detection model, illustrating an aluminum cylinder insonified by a dolphin click, whose waveform and power spectrum are also provided. The finite element mesh of the aluminum cylinder is shown on the right, with the axes in units of meters.

had a thickness of $6.35 \mathrm{~mm}$, with incremental changes, such as $-0.3 \mathrm{~mm}$, and $0.3 \mathrm{~mm}$, also being tested. All the cylinders had an outer diameter of $37.85 \mathrm{~mm}$. $\Delta \mathrm{TH}$ is defined as the wall thickness difference between the standard and comparison cylinders. Triangular elements were applied in this finite element model to allow for arbitrary shapes. The maximum mesh size $h$ was one-sixth of the wavelengths $(1.5 \mathrm{~mm}$ in water and $3.1 \mathrm{~mm}$ in aluminum). It makes the calculation error in an acceptable magnitude according to our recent study (Wei et al., 2018). The calculation error in the model is lower than $3.5 \%$ when the element sizes are smaller than one-fifth of the wavelength. The time-step used in the model was $9.865 \times 10^{-8} \mathrm{~s}$, which conformed to the CourantFriedrichs-Lewy (CFL) principle (de Moura and Kubrusly, 2013) that $\mathrm{CFL}=c \cdot \Delta t / h$ with $\mathrm{CFL}=0.2$, where $c$ was the sound velocity, $\Delta t$ was the time-step, and $h$ was the mesh size. The element order is quadratic and the basis function is a Lagrange function. On the outer edge of the water domain, the plane wave radiation was specified to allow outgoing waves to leave the modeling domain with minimal reflections. The simulation assumes that the sound waves propagate in infinite space. The convergence criterion of this model is that the iteration step is accepted if

$$
\left(\frac{1}{M} \sum_{j} \frac{1}{N} \sum_{i}\left(\frac{\left|E_{i} Y\right|}{A_{i}+R\left|Y_{i}\right|}\right)^{2}\right)^{1 / 2}<1,
$$

where $A_{i}$ is the absolute tolerance for the $i$ th degree of freedom, $M$ is the number of fields, $R$ is the relative tolerance, $N_{j}$ is the number of degrees of freedom in field $j$, and $Y$ is the solution vector. Both the relative tolerance and the absolute tolerance were set to the default values of 0.01 and 0.001 , respectively. Other parameters in the inequality were determined by the geometry, material, and mesh of the finite element model. No nonlinearities are involved since the applied physical equations and boundary conditions in this study are linear. For the simulations, parallel computing was used in a work station with two processors of Intel ${ }^{\circledR} \mathrm{Xeon}^{\circledR}$ CPU E5-2650 v2 2.60 GHZ (16 cores together).

As a typical case, the acoustic scattering field of a solid aluminum cylinder with normal plane wave incidence was analytically derived (Doolittle and Überall, 1966). The numerical solutions were in good agreement with the analytic solutions in both the frequency and space domain, and thus verified the reliability of the finite element simulations (Fig. 2).

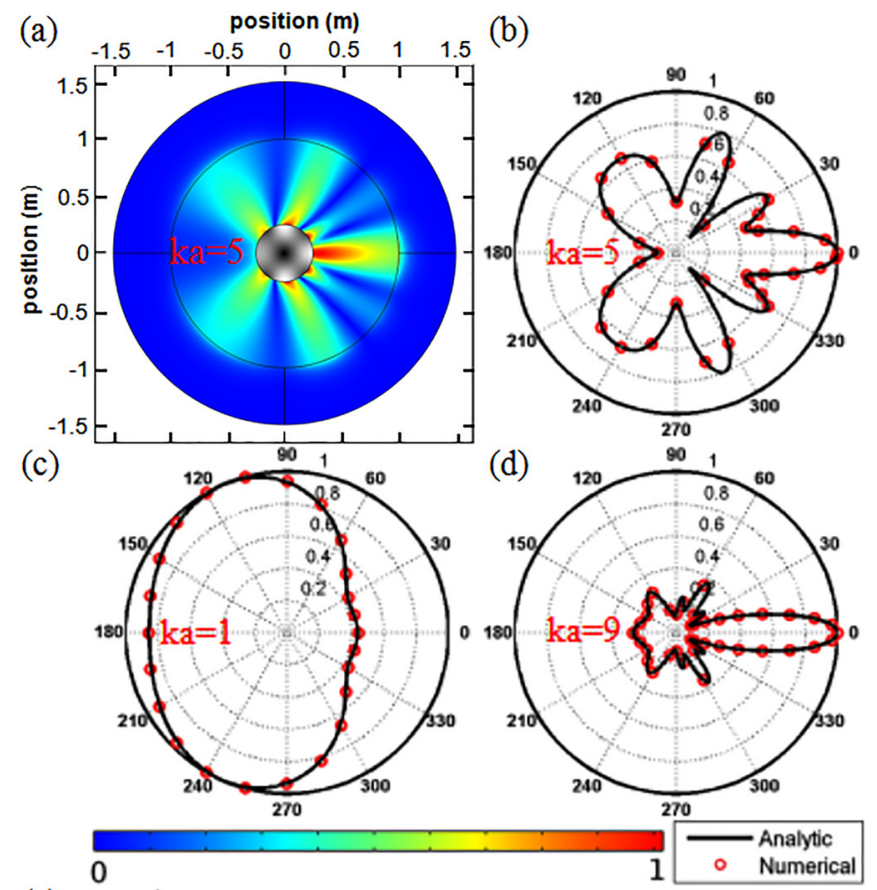

(e)

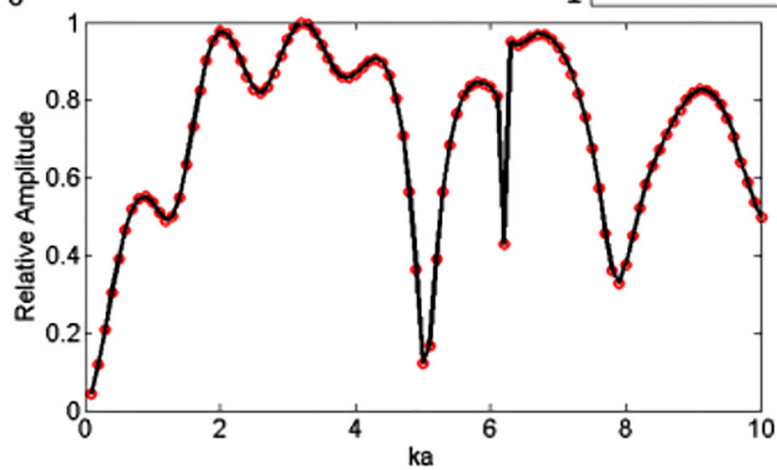

FIG. 2. (Color online) A comparison between the numerical and analytic solutions in both the frequency and space domains for the acoustic scattering of a solid aluminum cylinder with normal plane wave incidence. (a) The distribution of the sound intensity of the scattering field for ka $=5$, where ka is the product of the wavenumber $\mathrm{k}$ and the radius of solid cylinder a. (b)-(d) Directional beam patterns of the sound intensity of the scattering field for $\mathrm{ka}=5,1$, and 9. (e) The sound intensity of the scattering field in $180^{\circ}$ as a function of ka, where ka is a variable linear in frequency. 
Numerical results

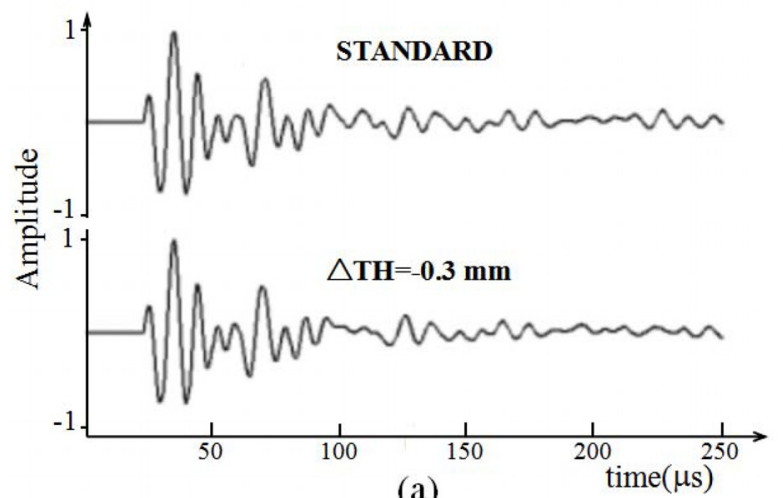

(a)
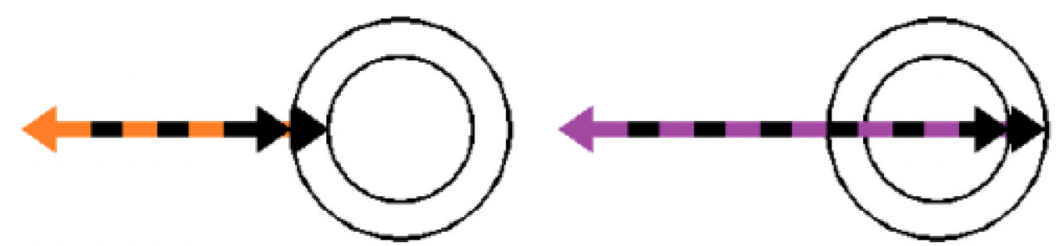

(c)

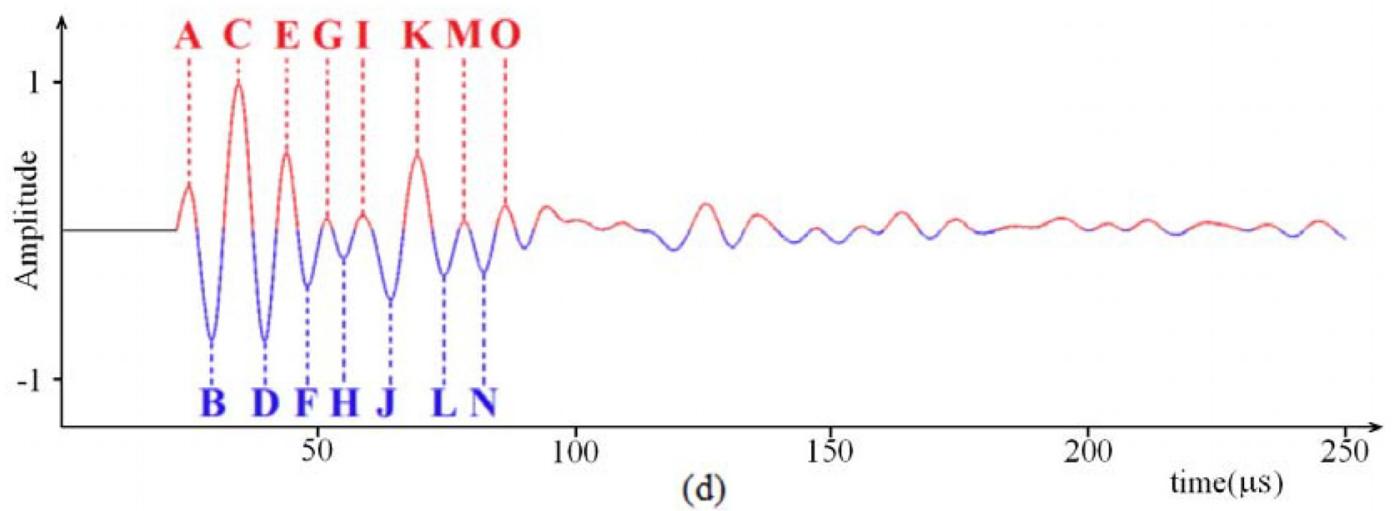

FIG. 3. (Color online) Acoustic echoes from the standard and $0.3 \mathrm{~mm}$ thinner cylinders, where (a) and (b) correspond to the numerical and experimental results. The experimental results are taken from Au and Pawloski (1992). The propagation paths of waves reflected from the front and rear walls are displayed (c). The positive peaks and negative troughs of the $-0.3 \mathrm{~mm}$ cylinder are distinguished with red and blue lines, respectively (d).

\section{RESULTS AND DICUSSIONS}

\section{A. Time and frequency comparisons with experimental measurements}

Figure 3 compares the simulated and measured echo waveforms of the standard cylinder and that $0.3 \mathrm{~mm}$ thinner, with the experimental results provided by $\mathrm{Au}$ and Pawloski (1992) being used for comparison. The amplitudes of the time domain numerical results were normalized with respect to the maximum value of the two thicknesses. The numerical results for the standard and comparison cylinder are superposed as noted in Fig. 5 to make the comparison easier. The corresponding frequency spectra comparisons are given in Fig. 4. Although the standard and $0.3 \mathrm{~mm}$ thinner cylinders had similar waveform and spectral patterns, their differences could be quantitatively extracted. In addition, the thinner cylinder led to resonances and even the whole spectrum shifting to a lower frequency band (Fig. 4). When the outer diameter of the cylinder remains unchanged, a thinner cylinder leads to a shorter propagation distance within the cylinder wall and a longer propagation distance in the water along the central path [Fig. 3(c)]. In this case, circumferential surface (Lamb) waves are likely to propagate more slowly in a thinner wall (Marston, 1989). Thus, the increased time interval between wave arrivals may lead to the spectrum shifting to a lower frequency band.

In experiments, it is difficult to accurately determine the real arrival times of echo peaks and troughs due to measurement errors and inevitable noise perturbations. In this paper, the echo peaks and troughs are numbered using upper case letters as in Fig. 3(d) and Fig. 6. This study provides a valuable method for determining the arrival times of echo peaks and troughs and allows comparisons of cylinders with different thicknesses at the same location. 
Numerical results

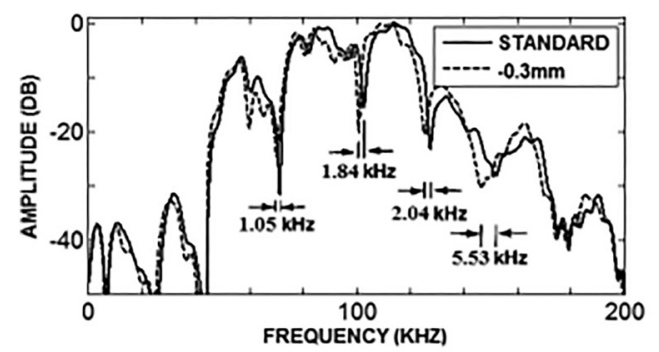

(a)

\section{Experimental results}

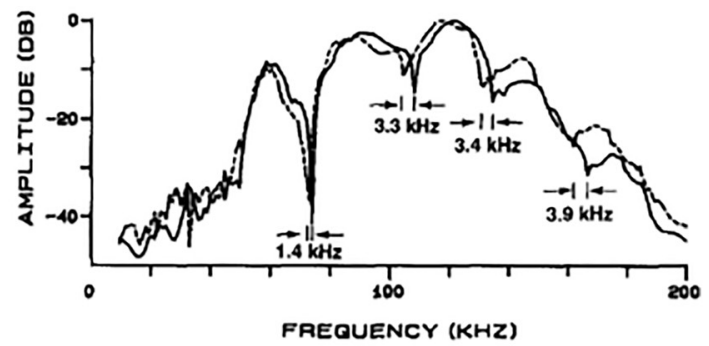

(b)

FIG. 4. Spectra of the acoustic echoes from the standard and $0.3 \mathrm{~mm}$ thinner cylinders, where the solid and dashed lines correspond to the standard and comparison cylinders, respectively. (a) and (b) correspond to the numerical and the experimental results, respectively. The experimental results are taken from Au and Pawloski (1992).

\section{B. Asymmetric cylinder wall thickness difference discrimination}

The finite element model was used to investigate the asymmetric cylinder wall thickness difference discrimination when the inner diameter was increased or decreased by the same amount of $\Delta \mathrm{TH}$. The acoustic echoes of the standard cylinder and the comparison cylinders with $\Delta \mathrm{TH}= \pm 0.3 \mathrm{~mm}$ are illustrated in Fig. 5(c). The returned echoes are from the

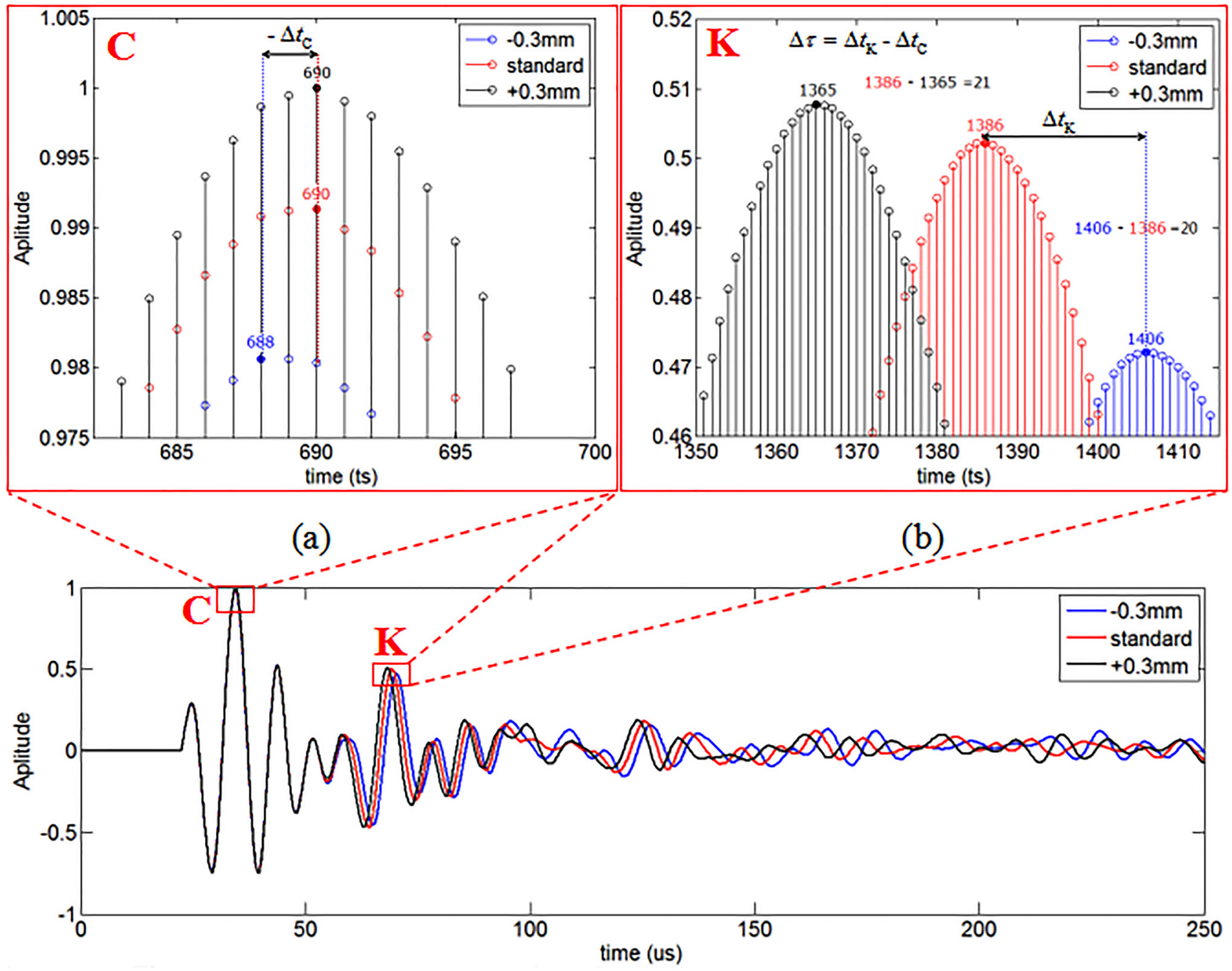

(c)

FIG. 5. (Color online) Comparisons of echoes from the cylinders with standard and $\pm 0.3 \mathrm{~mm}$ thicknesses (c), where the "C" and " $\mathrm{K}$ " peaks are zoomed out in (a) and (b), respectively. "ts" represents the sampling time interval. 


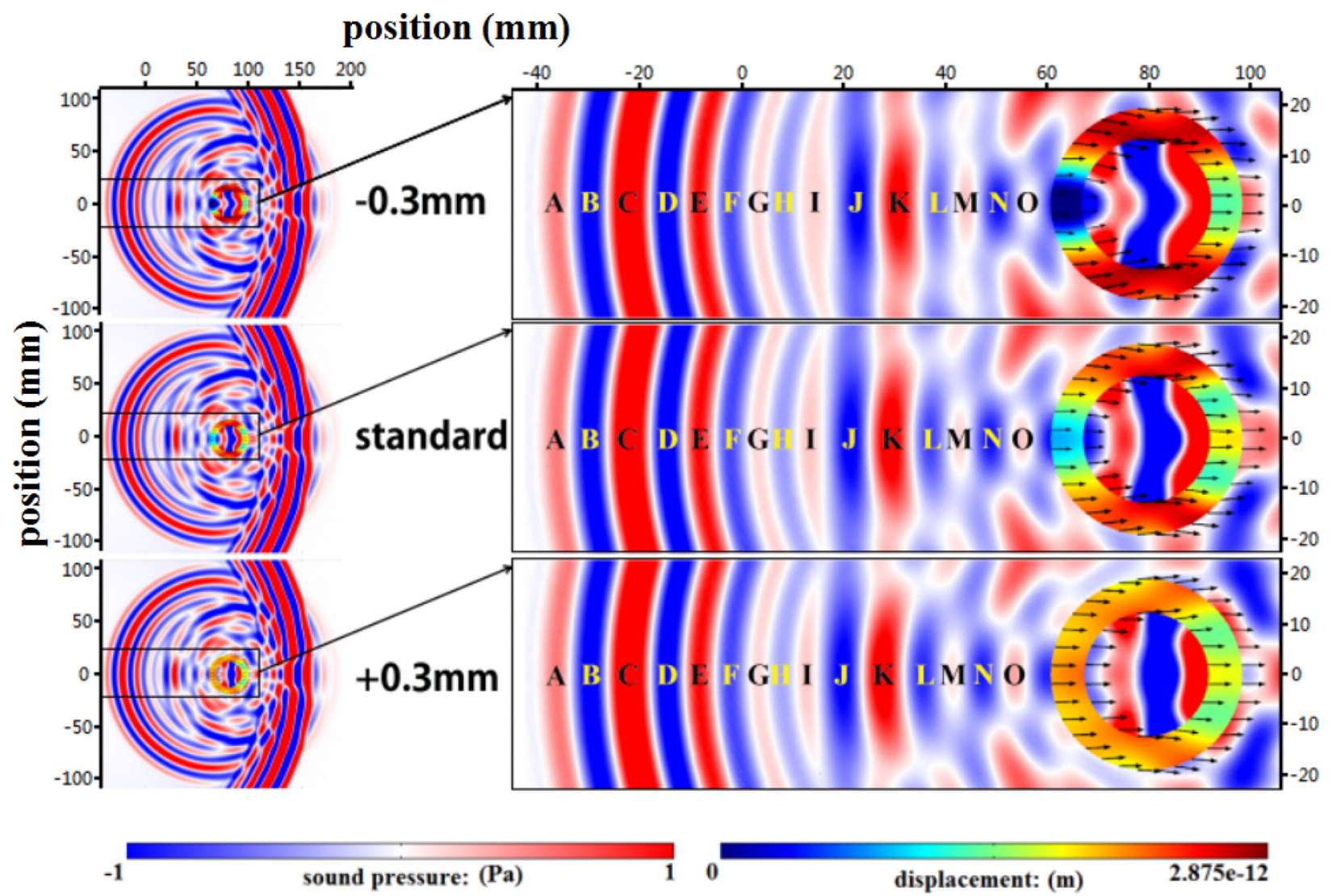

(a)

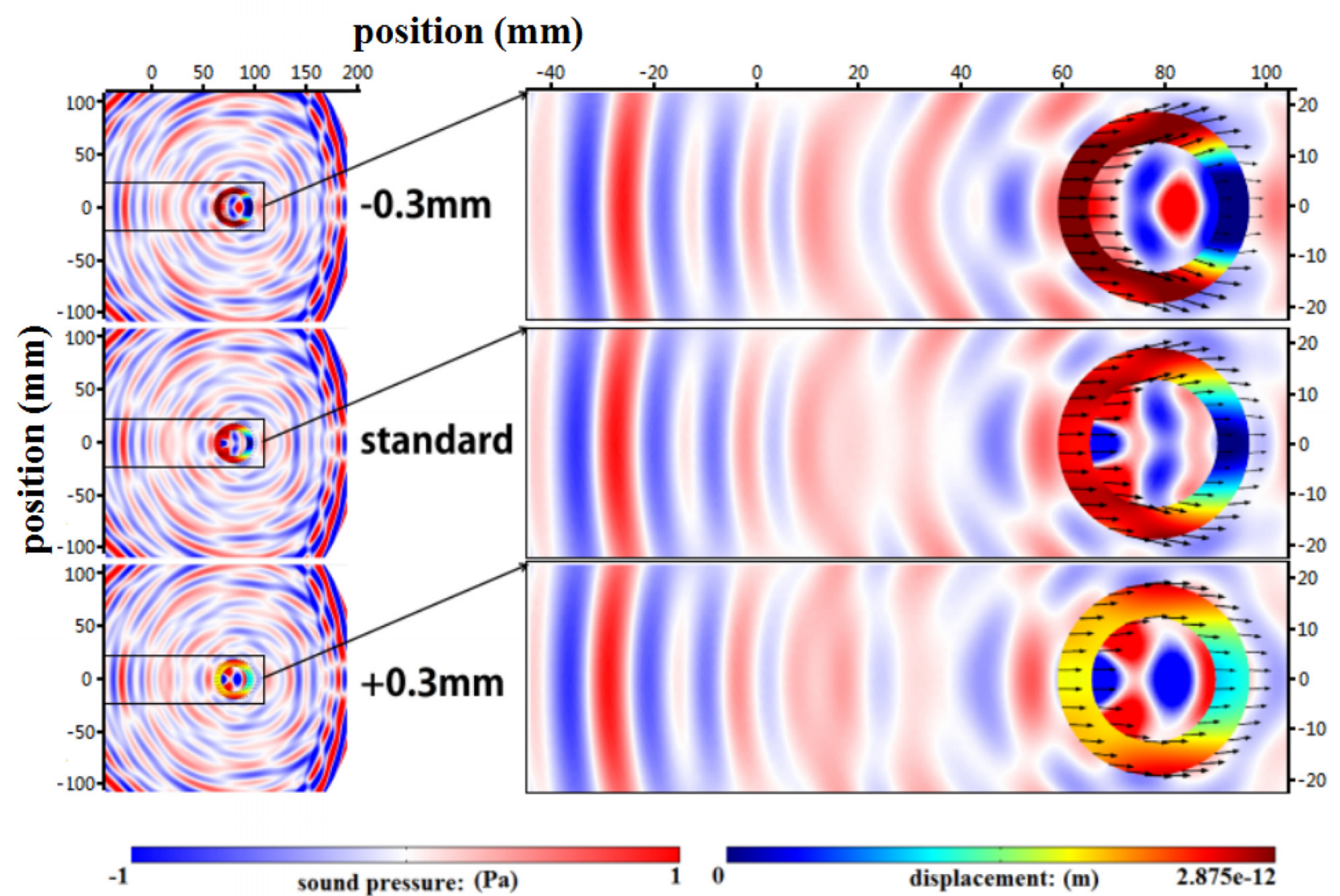

(b)

FIG. 6. (Color online) Spatial distributions of the sound pressures in the water and displacements in the standard and comparison cylinders, where (a), (b), and (c) correspond to $t=168.5 \mu \mathrm{s}, 205.6 \mu \mathrm{s}$, and $233.7 \mu \mathrm{s}$, respectively. At the bottom of panels (a), (b), and (c), the color scales for the sound pressure and displacement are presented. The arrows indicate both the directions and magnitudes of the displacements. 


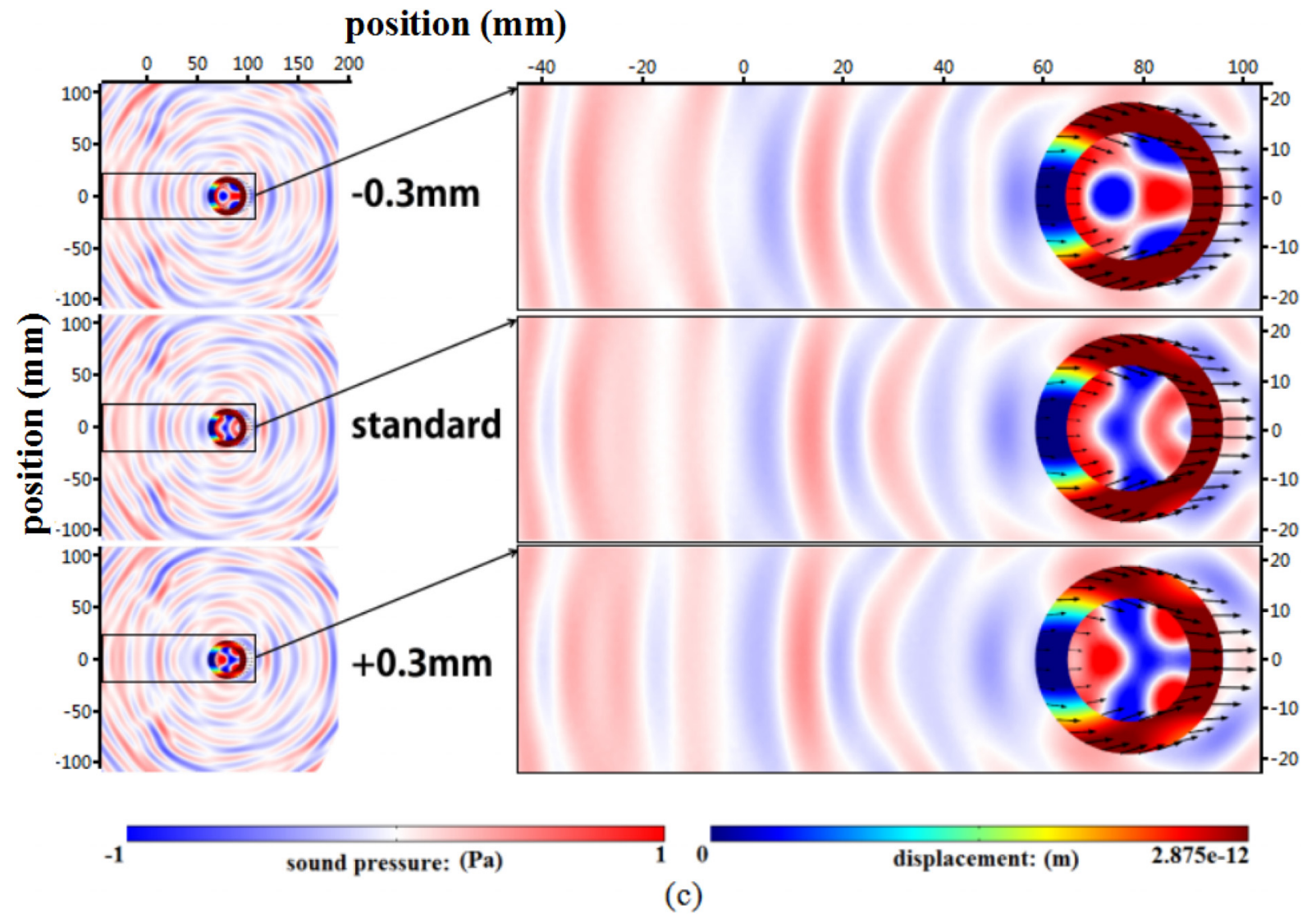

FIG. 6. (Color online) (Continued)

front edge of the shell walls. The reflection coefficients of the sound pressure going from water to aluminum and from aluminum to water are, respectively, 0.84 and -0.84 , where the negative sign designates a reversed phase. The wave arrivals from various paths created by reflection and transmission on interfaces, as well as the circumferential surface (Lamb) wave, contribute to the final returned echoes. Compared with the peaks nearby, the "C" and " $\mathrm{K}$ " peaks are the strongest [Figs. 5(a) and 5(b)]. The "C" peaks of the standard and $0.3 \mathrm{~mm}$ thicker cylinders both appear at the 690th sampling point, while that of the $0.3 \mathrm{~mm}$ thinner cylinder appears at the 688th sampling point [Fig. 5(a)]. Similarly, the arrival time shift of the " $\mathrm{K}$ " peak of the $0.3 \mathrm{~mm}$ thicker cylinder with respect to the standard cylinder is 21 sampling points, while that of the $0.3 \mathrm{~mm}$ thinner cylinder is 20 sampling points [Fig. 5(b)].

Based on geometric acoustics, the shift of the arrival time delay between the " $\mathrm{C}$ " and " $\mathrm{K}$ " peaks of comparison cylinder with respect to the standard cylinder is predicted as $\Delta \tau=\left|2 \Delta \mathrm{TH} / c_{l}-4 \Delta \mathrm{TH} / c_{0}\right| \quad(\mathrm{Au}$ and Pawloski, 1992), where $c_{0}$ is the sound velocity in water and $c_{l}$ is the longitudinal sound velocity in the cylinder wall. The shift of the arrival time delay between the " $C$ " and " $K$ " peaks of the $0.3 \mathrm{~mm}$ thinner cylinder with respect to standard cylinder $(\Delta \tau)$ is the difference between $\Delta t_{\mathrm{K}}$ and $\Delta t_{\mathrm{C}}$, that is, $\Delta \tau=\Delta t_{\mathrm{K}}-\Delta t_{\mathrm{C}}$, where $\Delta t_{\text {order }}$ represents the arrival time shift of echo peaks and troughs with the same sequence numbers [Figs. 5(a) and 5(b)]. For comparison with the wave acoustics obtained from the finite element simulation, the geometric acoustics and wave acoustics predicted values of $\Delta \tau$ are given in Table I. Geometric acoustics led the comparison cylinders with $\pm \Delta \mathrm{TH}$ thickness differences to have equal $\Delta \tau$ since $c_{0}$ and $c_{l}$ are constant. However, wave acoustics provides unequal $\Delta \tau$ values, as supported by the experimental measurement (Au and Pawloski, 1992). In addition, the thinner cylinder did have a slightly longer $\Delta \tau$ than the thicker one. The asymmetric arrival time shifts of the " $\mathrm{C}$ " and " $\mathrm{K}$ " peaks of the comparison cylinders with respect to the standard cylinder $\left(\Delta t_{\mathrm{C}}\right.$ and $\left.\Delta t_{\mathrm{K}}\right)$ are also given in Table II. This asymmetry of the arrival time shifts became more significant for echo peaks and troughs with higher sequence numbers [Fig. 5(c)].

When the acoustic waves arrive at the first interface of the cylinder, one component propagates back to the outside of the cylinder, while the other travels through the cylinder and arrives at the second interface. The same process occurs as the waves travel through the third and fourth

TABLE I. The shifts of the arrival time delay between the " $\mathrm{C}$ " and " $\mathrm{K}$ " peaks of the comparison cylinders with respect to the standard cylinder predicted by geometric acoustics and wave acoustics.

\begin{tabular}{lcc}
\hline \hline$\Delta \mathbf{T H}$ & $\Delta \tau$ (geometric acoustics) & $\Delta \tau$ (wave acoustics) \\
\hline $\mathbf{+ 0 . 3} \mathbf{~ m m}$ & $-0.72 \mu \mathrm{s}$ & $-1.05 \mu \mathrm{s}$ \\
$\mathbf{- 0 . 3 ~} \mathbf{~ m m}$ & $+0.72 \mu \mathrm{s}$ & $+1.10 \mu \mathrm{s}$ \\
\hline \hline
\end{tabular}


TABLE II. The arrival time shifts of the " $\mathrm{C}$ " and " $\mathrm{K}$ " peaks of the comparison cylinders with respect to the standard cylinder predicted by wave acoustics.

\begin{tabular}{|c|c|c|}
\hline$\Delta \mathrm{TH}$ & $\Delta t_{\mathrm{C}}$ & $\Delta t_{\mathrm{K}}$ \\
\hline$+0.3 \mathrm{~mm}$ & $0 \mu \mathrm{s}$ & $-1.05 \mu \mathrm{s}$ \\
\hline$-0.3 \mathrm{~mm}$ & $-0.1 \mu \mathrm{s}$ & $+1.00 \mu \mathrm{s}$ \\
\hline
\end{tabular}

interfaces. Under excitation from the sound waves, the cylinder vibrates in various modes and the existing modes superpose and interfere with each other. Since the first interface is very close to the second interface, the waves propagating between the first two interfaces superimpose and interfere with the specular reflected waves, so that the reflected waves from the front walls of cylinders with different thicknesses are not exactly the same [Fig. 5(a)]. A similar situation occurs at the third and fourth interfaces, thus affecting the envelopes of the waves reflected from the rear walls of the cylinders with different thicknesses [Fig. 5(b)]. Excitation clicks can be considered to be a linear combination (in fact, an integral) of elementary harmonic components. Since their wavelengths are comparable to the cylinder size (limited size of scatter), the group velocities of these elementary harmonic components of the cylinder are different and all lower than the intrinsic longitudinal sound velocity $c_{l}$ in the infinite medium. Therefore, the $\Delta \tau$ obtained from wave acoustics was larger than that from geometric acoustics (Table I).

Consider the four specular reflections from the cylinderwater interfaces, which are designated with the letters $\mathrm{W}-\mathrm{Z}$ : (W) the reflection from the outer surface of the front wall, (X) the reflection from the inner surface of the front wall, $(\mathrm{Y})$ the reflection from the inner surface of the rear wall, and (Z) the reflection from the outer surface of the rear wall. If we set the timing from reflection $\mathrm{W}$ to be zero, the time of each arrival is as follows:

$$
\begin{aligned}
& t_{W}=0, \\
& t_{X}=2 T H / c_{l}, \\
& t_{Y}=2 T H / c_{l}+4(a-T H) / c_{0}, \\
& t_{Z}=4 T H / c_{l}+4(a-T H) / c_{0},
\end{aligned}
$$

where $a$ is the cylinder's outer radius. The path timing differences between the standard and comparison cylinders will therefore be

$$
\begin{aligned}
\Delta t_{W} & =0, \\
\Delta t_{X} & =2(\Delta T H) / c_{l}, \\
\Delta t_{Y} & =2(\Delta T H) / c_{l}-4(\Delta T H) / c_{0}, \\
\Delta t_{Z} & =4(\Delta T H) / c_{l}-4(\Delta T H) / c_{0} .
\end{aligned}
$$

From these formulas, the timing differences are all linear in $\triangle T H$. Thus, the asymmetry observed is not caused by a superposition of these paths, since a superposition of a combination of these paths would also be linear in $\Delta T H$.
Therefore, the asymmetry cannot be explained by geometric acoustics.

Both the phase and group velocities of the circumferential surface (Lamb) wave on a curved shell have a nonlinear dependence on the cylinder wall thickness (Grigsby and Tajchman, 1961; Marston, 1989). According to the equations of propagation of Lamb waves (Grigsby and Tajchman, 1961), for infinite aluminum plates with standard thickness of $6.35 \mathrm{~mm}$ and $\pm 0.3 \mathrm{~mm}$ thickness variation, in the peak frequency of $120 \mathrm{kHz}$, the zeroth order asymmetric mode $A 0$ and the zeroth order symmetric mode $S 0$ are the existing modes and asymmetric variations in phase and group velocities were found. For the standard thickness, the phase and group velocities of $A 0$ mode are $2132.80 \mathrm{~m} / \mathrm{s}$ and $2985.05 \mathrm{~m} /$ $\mathrm{s}$, while those of $S 0$ mode are $5296.45 \mathrm{~m} / \mathrm{s}$ and $5168.45 \mathrm{~m} / \mathrm{s}$. For the $A 0$ mode, the asymmetric variations in phase velocity caused by $-0.3 \mathrm{~mm}$ and $+0.3 \mathrm{~mm}$ are $-30.46 \mathrm{~m} / \mathrm{s}$ and $28.77 \mathrm{~m} / \mathrm{s}$, while $-15.01 \mathrm{~m} / \mathrm{s}$ and $13.08 \mathrm{~m} / \mathrm{s}$ in group velocity. For the $S 0$ mode, the asymmetric variations in phase velocity caused by $-0.3 \mathrm{~mm}$ and $+0.3 \mathrm{~mm}$ are $5.82 \mathrm{~m} / \mathrm{s}$ and $-6.20 \mathrm{~m} / \mathrm{s}$, while $19.01 \mathrm{~m} / \mathrm{s}$ and $-20.38 \mathrm{~m} / \mathrm{s}$ in group velocity. This asymmetry or nonlinearity also exists in a curved shell (Marston, 1989). Therefore, the asymmetry in $\Delta \tau$ in the finite element model is associated with the circumferential surface (Lamb) wave in the cylinder wall.

Furthermore, the spatial distributions of the sound pressures and displacements of the standard cylinder and the comparison cylinders at $\mathrm{t}=168.5 \mu \mathrm{s}, 205.6 \mu \mathrm{s}$, and $233.7 \mu \mathrm{s}$ are compared in Figs. 6(a), 6(b), and 6(c), where the arrows indicate both the directions and magnitudes of the displacements. Including the specular reflected waves, elastic waves of various modes are generated to form a complex interference pattern. The wave pattern in space during a snapshot in time is similar to the time history of the wave passing through a single point in space, although the amplitude of the former further attenuates mainly due to wavefront expansion [Figs. 5(c) and 6]. In Fig. 6(a), for all cylinders, the echo peaks and troughs numbered as "A, B, C, D, E, and F" appear to have a similar and synchronous pattern, where " $\mathrm{C}$ " represents the strongest peak from the front wall as indicated in Fig. 3(d). The echo peaks and troughs labeled "I, J, K, L, M, N, and O" have obvious arrival time shifts, which is consistent with the comparison in the time domain in Fig. 5(c). Figures 6(a), 6(b), and 6(c) also verify the asymmetry in the arrival time shifts of the echo peaks and troughs and that the asymmetry becomes more significant for echo peaks and troughs with higher sequence numbers. At the same snapshot in time, the spatial distributions of the displacements in these cylinders with different thicknesses are different (Fig. 6). These finite element method derived data have higher resolution in both the time and space domains than those measured in the experimental tests and assist in understanding the mechanism of the dolphins' asymmetric cylinder wall thickness difference discrimination performance.

For an individual dolphin, differing performance can be attributed to differences in the input data. Dolphins discriminating more accurately when the cylinders were thinner likely benefited from reflected waves arriving later and thus 
being easier to differentiate. This may also be related to the broader backscattering process and the increasingly apparent asymmetry in the arrival time shifts of echo peaks and troughs and differences in the patterns of waveforms, which deserve further investigation. When performing target detection and discrimination, dolphins can adaptively adjust the characteristics of their click trains to better perceive the surrounding environment. For example, in one experiment, a harbour porpoise used clicks of lower energy and frequency when investigating a larger sphere (Teilmann et al., 2002). Nevertheless, the asymmetry property has proved intrinsic and universal by wave acoustics, as determined by the circumferential surface (Lamb) waves. Dolphins behave like an energy detector with an integration time of $264 \mu \mathrm{s}$ (Au et al., 1988). Both energy and arrival time differences can be extracted by dolphins to perform target discrimination. The integration may be associated with perception, while the arrival time differences may be associated with the physical process of acoustic scattering. Dolphins use multiple features from the echoes to achieve better performance (DeLong et al., 2006). Finally, previous studies on biosonar and biomimetic sonar have focused on sound transmission, especially in terms of the excitation waveform design (Capus et al., 2007) and directivity along with related manipulation or adjustment mechanisms (Zhang et al., 2017) and on receiving (Dobbins, 2007). Methods for modeling underwater target detection and discrimination by dolphins were rarely involved. The traditional method used in technical sonar to discriminate cylinder wall thickness differences is to compare the time delay between reflected waves. The asymmetric discrimination accuracy demonstrated here along with associated features of the broad backscattering process could provide inspiration to fields from biosonar to biomimetic sonar to fully utilize information from the whole process, allowing an enhanced resolution in the thickness and improved anti-noise techniques.

\section{CONCLUSIONS}

In this paper, the asymmetric cylinder wall thickness difference discrimination by dolphins was investigated using an underwater target detection model based on the finite element method. The model was verified to be reliable by comparing the simulated acoustic scattering field of a solid elastic cylinder with its analytic solution. Qualitative consistency between the numerical and experimental results was found in both the echo waveforms and frequency spectra, further suggesting the feasibility of this proposed underwater target detection and discrimination model. The thinner cylinder shifted the spectrum to a lower frequency band, which was associated with longer arrival time delays. Asymmetric arrival time shifts of the echo peaks and troughs with the same sequence numbers along with asymmetric shifts of the arrival time delay between echo peaks and troughs for comparison cylinders with thickness changes in opposite directions were also found, as measured by $\mathrm{Au}$ and Pawloski (1992). The asymmetry became more significant for echo peaks and troughs with higher sequence numbers. Apart from the asymmetric arrival time shifts of the acoustic echoes, the patterns of the echo waveforms, the spatial distributions of the sound pressures in the water, and particle vibratory displacements in the cylinders also varied with the cylinder thickness. Its physical origin was explored using both geometric acoustics and wave acoustics. The asymmetry observed might be caused by the circumferential surface (Lamb) wave in the cylinder wall, which is a wave acoustics phenomenon that cannot be derived from geometric acoustics. Our findings might be valuable not just for understanding the mechanism of the effect described, but also for helping the development of biomimetic intelligence for robust signal processing in underwater target discrimination.

\section{ACKNOWLEDGMENTS}

This work is financially supported by the National Natural Science Foundation of China (Grant Nos. 41276040, 41676023, and 11174240), the Natural Science Foundation of Fujian Province of China (Grant No. 2012J06010), and the Fundamental Research Funds for the Central Universities (Grant No. 20720180123). The work is also sponsored by the Scientific Research Foundation for the Returned Overseas Chinese Scholars, State Education Ministry. We thank Professor Whitlow Au for use of experimentally measured results in Figs. 3(b) and 4(b). We are grateful to Xiaohui Xu for his assistance.

Aroyan, J. L. (2001). "Three-dimensional modeling of hearing in Delphinus delphis," J. Acoust. Soc. Am. 110(6), 3305-3318.

Aroyan, J. L., Cranford, T. W., Kent, J., and Norris, K. S. (1992).

"Computer modeling of acoustic beam formation in Delphinus delphis," J. Acoust. Soc. Am. 92(5), 2539-2545.

Au, W. W., Andersen, L. N., Rasmussen, A. R., Roitblat, H. L., and Nachtigall, P. E. (1995). "Neural network modeling of a dolphin's sonar discrimination capabilities," J. Acoust. Soc. Am. 98(1), 43-50.

Au, W. W., and Hastings M C. (2008). Principles of Marine Bioacoustics (Springer-Verlag, New York), pp. 515-521, 534-535.

Au, W. W., Moore, P. W., and Pawloski, D. A. (1988). "Detection of complex echoes in noise by an echolocating dolphin," J. Acoust. Soc Am. 83(2), 662-668.

Au, W. W., and Pawloski, D. A. (1992). "Cylinder wall thickness difference discrimination by an echolocating Atlantic bottlenose dolphin," J. Compar. Physiol. A 170(1), 41-47.

Au, W. W. L., and Penner, R. H. (1981). "Target detection in noise by echolocating Atlantic bottlenose dolphins," J. Acoust. Soc. Am. 70(3), 687-693.

Au, W. W. L., and Snyder, K. J. (1980). "Long-range target detection in open waters by an echolocating Atlantic Bottlenose dolphin (Tursiops truncatus)," J. Acoust. Soc. Am. 68(4), 1077-1084.

Au, W. W. L., and Turl, C. W. (1983). "Target detection in reverberation by an echolocating Atlantic bottlenose dolphin (Tursiops truncatus)," J. Acoust. Soc. Am. 73(5), 1676-1681.

Au, W. W. L., and Turl, C. W. (1991). "Material composition discrimination of cylinders at different aspect angles by an echolocating dolphin," J. Acoust. Soc. Am. 89(5), 2448-2451.

Brill, R. L., Pawloski, J. L., Helweg, D. A., Au, W. W., and Moore, P. W. (1992). "Target detection, shape discrimination, and signal characteristics of an echolocating false killer whale (Pseudorca crassidens)," J. Acoust. Soc. Am. 92(3), 1324-1330.

Busnel, R. G., and Fish, J. F. (1980). Animal Sonar Systems (SpringerVerlag, Boston, MA), pp. 71-95.

Capus, C., Pailhas, Y., Brown, K., Lane, D. M., Moore, P. W., and Houser, D. (2007). "Bio-inspired wideband sonar signals based on observations of the bottlenose dolphin (Tursiops truncatus)," J. Acoust. Soc. Am. 121(1), 594-604.

Delong, C. M., Au, W. W., Lemonds, D. W., Harley, H. E., and Roitblat, H. L. (2006). "Acoustic features of objects matched by an echolocating bottlenose dolphin,” J. Acoust. Soc. Am. 119(3), 1867-1879. 
de Moura, C. A., and Kubrusly, C. S. (2013). The Courant-Friedrichs-Lewy (CFL) Condition: 80 Years After Its Discovery (Birkhäuser, Basel), pp. $1-234$.

Dobbins, P. (2007). "Dolphin sonar-Modelling a new receiver concept," Bioinspir. Biomimet. 2(1), 19-29.

Doolittle, R. D., and Überall, H. (1966). "Sound scattering by elastic cylindrical shells," J. Acoust. Soc. Am. 39(2), 272-275.

Gao, X., Zhang, Y., Cao, W., Dong, E., Song, Z., Li, S., Tang L., and Zhang, S. (2016). "Acoustic beam control in biomimetic projector via velocity gradient," Appl. Phys. Lett. 109(1), 013505.

Gaunaurd, G. C., Brill, D., Huang, H., and Moore, P. W. (1996). "Ultrasonic spectroscopy techniques used by dolphins to characterize resonating submerged elastic shells," J. Acoust. Soc. Am. 99(4), 2581-2603.

Grigsby, T. N., and Tajchman, E. J. (1961). "Properties of Lamb waves relevant to the ultrasonic inspection of thin plates," IRE Trans. Ultrason. Eng. 8(1), 26-33.

Helweg, D. A., Au, W. W., Roitblat, H. L., and Nachtigall, P. E. (1996). "Acoustic basis for recognition of aspect-dependent three-dimensional targets by an echolocating bottlenose dolphin," J. Acoust. Soc. Am. 99(1), 2409-2420.

Marston, P. L. (1989). "Phase velocity of Lamb waves on a spherical shell: Approximate dependence on curvature from kinematics," J. Acoust. Soc. Am. 85(6), 2663-2665.

Moore, A. P. W. (1997). "Mine-hunting dolphins of the Navy," SPIE Proc. 3079, 2-6.

Moore, P. W., Dankiewicz, L. A., and Houser, D. S. (2008). "Beamwidth control and angular target detection in an echolocating bottlenose dolphin (Tursiops truncatus)," J. Acoust. Soc. Am. 124(5), 3324-3332.

Moore, P. W., Roitblat, H. L., and Nachtigall, P. E. (1993). "Recognizing successive dolphin echoes with an integrator gateway network," in Proceedings of the 27th Asilomar Conference on Signals, Systems and Computers, 1-3 November 1993, Pacific Grove, CA (IEEE, New York), Vol. 1, pp. 588-592.

Müller, R., and Kuc, R. (2007). "Biosonar-inspired technology: Goals, challenges and insights," Bioinspir. Biomimet. 2(4), S146-S161.

Pack, A. A., and Herman, L. M. (1995). "Sensory integration in the bottlenosed dolphin: Immediate recognition of complex shapes across the senses of echolocation and vision," J. Acoust. Soc. Am. 98(2), 722-733.
Pailhas, Y., Capus, C., Brown, K., and Moore, P. (2010). "Analysis and classification of broadband echoes using bio-inspired dolphin pulses," J. Acoust. Soc. Am. 127(6), 3809-3820.

Qiao, G., Qing, X., Feng, W., Liu S., Nie, D., and Zhang, Y. (2017). "Elastic feature of cylindrical shells extraction in time-frequency domain using biomimetic dolphin click," J. Acoust. Soc. Am. 142(6), 3787-3795.

Song, Z., Zhang, Y., Wei, C., and Wang, X. (2016). "Inducing rostrum interfacial waves by fluid-solid coupling in a Chinese river dolphin (Lipotes vexillifer)," Phys. Rev. E 93(1), 012411.

Teilmann, J., Miller, A., Kastelein, R., Madsen, P., Nielsen, B., and Au, W. W. (2002). "Characteristics of echolocation signals used by a harbour porpoise (Phocoena phocoena) in a target detection experiment," Aquatic Mammals 28(3), 275-284.

Turl, C. W., Penner, R. H., and Au, W. W. (1987). "Comparison of target detection capabilities of the beluga and bottlenose dolphin," J. Acoust. Soc. Am. 82(5), 1487-1491.

Turl, C. W., Skaar, D. J., and Au, W. W. (1991). "The echolocation ability of the beluga (Delphinapterus leucas) to detect targets in clutter," J. Acoust. Soc. Am. 89(2), 896-901.

Wei, C., Song, Z., Au, W. W., Zhang, Y., and Wang, D. (2018). “A numerical evidence of biosonar beam formation of a neonate Yangtze finless porpoise (Neophocaena asiaeorientalis)," J. Theor. Comput. Acoust. 26(2), 1850009.

Wei, C., Zhang, Y., and Au, W. W. (2014). "Simulation of ultrasound beam formation of baiji (Lipotes vexillifer) with a finite element model," J. Acoust. Soc. Am. 136(1), 423-429.

Wezensky, E. M., Finneran, J. J., Mulsow, J., Martin, C. R., Branstetter, B. K., Moore, P. W., and Houser, D. S. (2011). "Performance of an echolocating bottlenose dolphin in the presence of anthropogenic masking noise," J. Acoust. Soc. Am. 130(4), 2561.

Zaitseva, K. A., and Korolev, V. I. (2005). "On the role of echosignal temporal microstructure in mechanisms of classification of small-size targets by Dolphins Tursiops truncatus," J. Evol. Biochem. Physiol. 41(4), 456-464.

Zhang, Y., Gao, X., Zhang, S., Cao, W., Tang, L., Wang, D., and Li, Y. (2014). "A biomimetic projector with high subwavelength directivity based on dolphin biosonar," Appl. Phys. Lett. 105(12), 123502.

Zhang, Y., Song, Z., Wang, X., Cao, W., and Au, W. W. (2017). "Directional acoustic wave manipulation by a porpoise via multiphase forehead structure," Phys. Rev. Appl. 8(6), 064002. 